\title{
Dual channel self-oscillating optical magnetometer
}

\author{
J. Belfi ${ }^{1,} 3$, G. Bevilacqua, ${ }^{1}$ V. Biancalana, ${ }^{1, *}$ S. Cartaleva, ${ }^{2}$ Y. Dancheva, ${ }^{1}$ K. Khanbekyan ${ }^{1}$ \\ and L. Moi ${ }^{1}$ \\ ${ }^{1}$ CNISM-Unità di Siena, Centro Studi Sistemi Complessi and Dipartimento di Fisica - Università di \\ Siena, via Roma 56, 53100 Siena, Italy \\ ${ }^{2}$ Institute of electronics, BAS, Boul. Tsarigradsko Shosse 72, 1784 Sofia, Bulgaria \\ *Corresponding author: biancalana@unisi.it
}

\begin{abstract}
We report on a two-channel magnetometer based on nonlinear magneto-optical rotation in a Cs glass cell with buffer gas. The Cs atoms are optically pumped and probed by free running diode lasers tuned to the $\mathrm{D}_{2}$ line. A wide frequency modulation of the pump laser is used to produce both synchronous Zeeman optical pumping and hyperfine repumping. The magnetometer works in an unshielded environment and spurious signal from distant magnetic sources is rejected by means of differential measurement. In this regime the magnetometer simultaneously gives the magnetic field modulus and the field difference.

Rejection of the common-mode noise allows for high-resolution magnetometry with a sensitivity of $2 \mathrm{pT} / \sqrt{\mathrm{Hz}}$. This sensitivity, in conjunction with long-term stability and a large bandwidth, makes possible to detect water proton magnetization and its free induction decay in a measurement volume of $5 \mathrm{~cm}^{3}$. (C) 2018 Optical Society of America
\end{abstract}

OCIS codes: $020.1670,230.1150$

\section{Introduction}

The potential of atomic magnetometers is well known and was established in the late 50's and the 60's [1-5]

There has been a recent revival of the interest in such devices, specifically in optical atomic magnetometers, also due to the progress made in diode laser technology. This revival has led to the achievement of impressive results over the last decade [6-9], highlighting the real applicability of the optical magnetometry due to its high sensitivity, high accuracy, and high time and spatial resolution (see also the review [10] and the references therein for a panorama on this progresses). For each particular application, a different set of specifications have to be stressed to their extreme limit, and atomic magnetometers are excellent in terms of trade off flexibility, when seeking appropriate compromises. Optical magnetometry based on Faraday rotation has recently been demonstrated to be an impressively powerful tool even in dense media such as liquids [11], where optical rotation directly induced by the nuclear spin polarization has been observed.

The growing interest in this field is also specifically related to the application of optical magnetometers as non-inductive detectors of low-field nuclear magnetic resonance (NMR) detection and to the close area of its remote detection [12], as an alternative to the superconducting quantum interference device approach. Low-field NMR imaging with $100 \mathrm{msec}$ and millimetre size resolution has been demonstrated [13], and the impressive limit of $7 \mathrm{fT} / \sqrt{\mathrm{Hz}}$ has been achieved with a NMR detector [14] operating with Potassium in

\footnotetext{
${ }^{3}$ presently at: Dipartimento di Fisica, Università di Pisa, Largo B. Pontecorvo 3, 56127 Pisa, Italy
} 
the $100 \mathrm{kHz}$ range. A miniaturized (chip-scale) atomic magnetometer has been developed, working in spinexchange relaxation-free regime, which can detect the proton field from Hydrogen contained in $1 \mathrm{~mm}^{3}$ water prepolarized at $1 \mathrm{~T}$ field, with measuring time of $1 \mathrm{sec}$ [15].

In the last few years, our research has been devoted to the construction of magnetometric setups, considering their possible practical applications and in-field use [16]. Our goal is to build simple but reliable setups with good sensitivity and long-term stability, operating at room temperature, in an unshielded room, and using commercially available laser sources. The challenge of operating in an unshielded environment has led us to develop differential setups [17]. We have chosen $\mathrm{Cs}_{2}$ line for operation at room temperature with commercial lasers. This compromise leads to disadvantages related to the smaller Landé factor and to lower efficiency in the pumping process, but the advantage of a single isotope, high density medium at room (or slightly higher) temperature.

The flexibility of the setup presented here is related to the presence of two independent light sources, which makes it possible to precisely determine the optimal optical detuning of the probe laser. This configuration is also suitable for analyzing and studying subjects of general and basic research, such as the collisional properties of the excited states, the interplay of hyperfine and Zeeman pumping etc. However, these subjects are beyond the scope of this paper.

Light generally plays a double role in the optical magnetometers [18], both creating atomic momentum orientation/alignment and detecting its time evolution. This evolution (atomic momentum precession around the magnetic field to be measured) is as free as the perturbation produced by the probing light is negligible.

Frequency or amplitude modulation of the laser radiation are widely used to prepare the state at given instants, and to detect the consequent optical properties of the medium, which are time-dependent due to the coherently precessing atomic spins. These properties can be characterized in terms of absorptive, dispersive and dichroic behaviour.

The external degrees of freedom of the atoms (translational motion) may play a dominant role in degrading the sensitivity of an atomic magnetometer by reducing of the interaction time of atoms - either bringing them out of the interaction volume, or by causing them to collide destructively with the cell walls. Buffer gas [19] and anti-relaxation coatings have been widely and successfully used for decades now as a solution to these problems. The coating approach demands the production of very high quality surface covering and a few groups around the world $[6,20,21]$ have developed reliable procedures, with which excellent results have been achieved. Recently, J. M. Higbie et al. [22] built a robust self-oscillating setup based on high quality antirelaxation paraffin-coated cells excited by two non-overlapping parallel beams.

Aiming to build a robust self-oscillating magnetometer with a buffer gas cell, we developed a setup similar to the one described in [22], but using two laser beams crossing inside the cell, as diffusionally slowed motion inside the buffer gas would make it impossible to pump and detect atoms in different locations. In fact, in our experimental conditions (90 Torr of Ne as a buffer gas) the diffusion coefficient of Cs can be estimated (see e.g. [23]) as being about $1 \mathrm{~cm}^{2} / \mathrm{s}$. This leads, within a typical coherence time (in the range $10 \div 100 \mathrm{~ms}$ ), to typical atomic displacements of $1 \div 3 \mathrm{~mm}$ - an amount of the same order as the beam size.

The use of buffer gas reduces the efficiency of the Zeeman pumping process, compared to hyperfine pumping, due to the stirring effect of collisions between excited alkali atoms and buffer gas atoms. This effect leads to higher hyperfine pumping with respect to Zeeman pumping and thus to a significant lack of signal, unless appropriate repump radiation is used. This is what actually occurs in our setup, where a low-power wide frequency modulated beam synchronously induces Zeeman optical pumping and counteracts the hyperfine optical pumping while an unmodulated probe beam detects the atomic spin precession.

The use of two separate beams makes it easy to operate the whole system as a self-oscillator, considerably widening the response bandwidth with respect to the scanned regime [24] and thus also making it an attractive 
tool in fields of application that demand a fast response, such as the detection of oscillating fields produced by nuclear precession in low-field NMR experiments.

In our case the two beams are generated by two independent laser sources, in contrast with the alternative approach based on splitting an unmodulated beam and then passively modulating one of the two beamlets, as done in the recent experiments of P. D. D. Schwindt et al. [25] and J. M. Higbie et al. [22]).

As described in the next sections, our magnetometer can operate both with a single sensor, or with a couple of sensors with a differential response, making simultaneous absolute and differential magnetometry possible, with the advantage of significant common-mode noise rejection. The latter is of a great importance when measuring slowly varying magnetic fields in an unshielded environment.

\section{Experimental Setup}

The interaction scheme is suitable for a transverse pumping experiment, i.e. for measurement of a magnetic field (oriented along the $z$ axis) that is perpendicular to the laser beam propagation ( $y$ direction) and hence to the laser-induced orientation of the atomic momentum. The experimental apparatus, shown schematically in Fig प consists of two cylindrical Cs cells of $2 \mathrm{~cm}$ in diameter and length, crossed by two laser beams the pump and the probe beam. The Cs cells are filled with 90 Torr of Ne and kept at around $32^{\circ} \mathrm{C}$ using

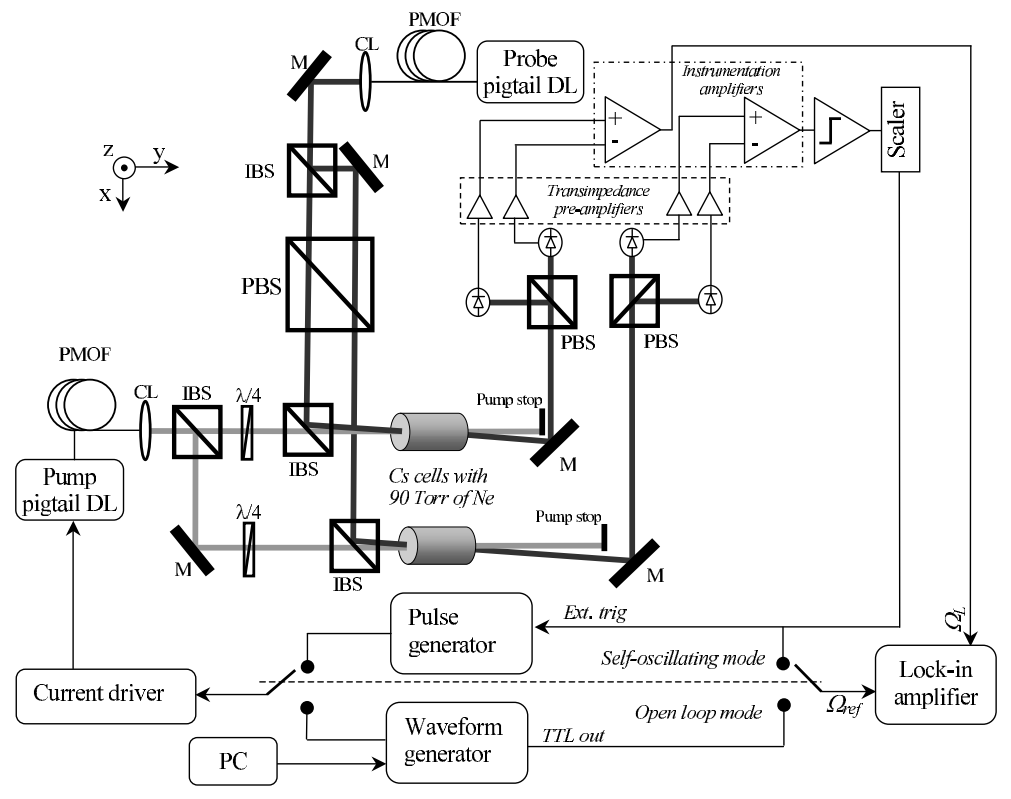

Fig. 1. Schematic of the experimental setup. Abbreviations: PMOF: Polarization Maintaining Optical Fiber, PBS: Polarizing Beam Splitter, IBS: intensity beam splitter, M: mirror, CL: collimating lens, $\lambda / 4$ : quarter-wave plates.

circulating hot water. The probe laser is $1.9 \mathrm{GHz}$ blue-shifted from the transition starting from $F_{g}=4$. The two laser beams, tuned to the Cs $D_{2}$ line, are supplied by two pigtail diode lasers in free running regime (UniPhase-SDL laser chip (5411-G1-852), a Faraday isolator (40 dB) and polarization maintaining fiber assembled by HIV, Germany). The pump laser frequency is passively stabilized and $3 \mathrm{GHz}$ red-shifted from the transition starting from $F_{g}=3$ and is widely modulated around this frequency position by applying appropriate pulses at the modulation input of the current driver. 
The pump and the probe beams pass through each cell at a small angle to each other so that they can be separated at the detection stage (around $2 \mathrm{~m}$ away), before which the pump beam is blocked. Each probe beam, linearly polarized in the $x$ direction, is analyzed after the cell using a balanced polarimeter. Each polarimeter is composed of a Wollaston polarizing beam splitter (PBS) oriented at $45^{\circ}$ with respect to the $z$ and $x$ axes. The photodiode currents are amplified by two pairs of identical transimpedance preamplifiers with maximum amplification at around $15 \mathrm{kHz}$ and a bandwidth of about $17 \mathrm{kHz}$ [26]. The outputs of each pair of transimpedance amplifiers are fed into an instrumentation amplifier (AD620) whose output is monitored with an oscilloscope or acquired. The signal from the main arm is also used to close the selfoscillating loop (to trigger the pulses applied to the pump laser driver) as well as for referencing a lock-in amplifier.

\section{Detection principle}

A detailed representation of the excitation/detection scheme is given in Fig 22 The probe beam is left unmodulated, while the pump beam is modulated by applying a pulse sequence at a frequency $\nu$ and a duty cycle of $30 \%$ to the modulating input of the diode current driver. Due to the limited bandwidth of the modulation input (the nominal cutoff frequency is $25 \mathrm{kHz}$ ), some distortion of the pulsed waveform occurs. Fig 2 shows the absorption profile of the set of 6 possible transitions for homogeneous broadening due to high buffer gas pressure, and without considering the hyperfine optical pumping effect. The center of each transition is marked, together with its relative oscillator strength. The probe beam is blue-detuned with respect to the group of transitions starting from $F_{g}=4$, while the pump beam is broadly modulated, with an estimated frequency deviation of the order of $14 \mathrm{GHz}$. The modulation duty cycle and detuning of the pump laser make it resonant for short time intervals (pulses) with $\mathrm{F}_{g}=4$ and for longer time intervals with $\mathrm{F}_{g}=3$. The presence of two laser sources makes it possible to identify the optimal optical detuning of each one. The probe laser detuning is important in terms of resonance contrast and linewidth. The optimal optical detuning of the probe laser is found in scanned mode, by looking at the slope of the resonance curve expressed as $\mathrm{A} / \gamma^{3}$, where the parameters $\mathrm{A}$ and $\gamma$ are the amplitude and the width of the resonance profile, respectively, and are determined by means of a best fit procedure with a Lorentzian curve. The slope decreases asymmetrically around the maximum at $1.9 \mathrm{GHz}$ and goes down to $80 \%$ at 1.2 and $4.9 \mathrm{GHz}$ blue-detuning from $F_{g}=4$ group of lines. The optical frequency of the probe laser is passively stabilized at the optimal value, using the Doppler broadened absorption profile observed in the transmission of a vacuum Cs cell as a reference. This passive stabilization is described in detail in [24] and provides long term drift correction unless mode-hop occurs, with an uncertainty of $2 \mathrm{MHz}$ for time scales of $1 \mathrm{sec}$. The wide frequency modulated pump laser is left unstabilized, with optical frequency excursions of the order of $200 \mathrm{MHz}$ per day. An optical frequency drift of $700 \mathrm{MHz}$ from the optimal set point gives a decrease of the resonance slope of the order of $20 \%$.

The (circularly polarized) pump beam produces Zeeman optical pumping by orienting the population orthogonally to the bias magnetic field. After a pump pulse, the atomic spins undergo Larmor precession at frequency $\nu_{L}$. The probe beam experiences rotation of the polarization, whose amount depends on time via the Larmor precession angle. If the optical pumping rate is modulated at frequency $\nu$, then the optical-rotation angle of the probe polarization will, in general, also oscillate at $\nu$. The rotation amplitude is maximum, provided that the pump pulses are synchronous with the Larmor precession, i.e. if $\nu$ matches $\nu_{L}$ or its sub-

harmonics. In the results presented here we force the system with the first sub-harmonic, i.e. a pump pulse is applied for each couple of Larmor cycles. The rotation signal, detected at the balanced polarimeter and shown in Fig [ is essentially sinusoidal at the Larmor frequency, which makes it suitable to trigger the pump pulses in the self-oscillating mode without any filtering in the loop [25]. The total harmonic distortion (ratio between harmonics and fundamental tone amplitudes) is about $2.2 \%$, including the integer and half-integer 


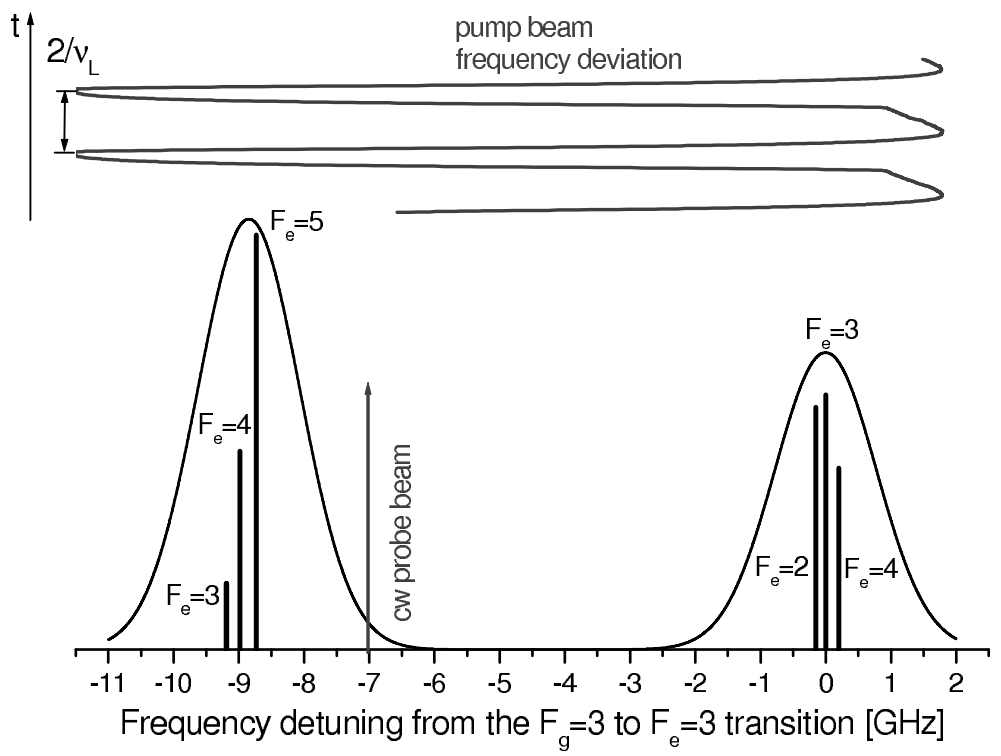

Fig. 2. Instantaneous optical frequency of the pump laser with respect to the absorption profile of the $\mathrm{Cs}_{2}$ line. The distortion of the square wave that modulates the pump laser frequency is inferred by direct measurement of the driving current.

harmonics that originate from stray light from the pump laser (see trace d in Fig 3). The SINAD, i.e. the ratio between the fundamental tone and the residual (noise+harmonics) in the bandwidth of the transimpedance amplifier, is $31 \mathrm{~dB}$, corresponding to a ratio of 36 between the rms amplitude of the fundamental tone and the residual. The fundamental amplitude of $\pm 1.6 \mathrm{~V}_{p-p}$ corresponds to $\pm 4 \mathrm{mrad}$ optical rotation, so that the measured residual corresponds to an uncertainty of $80 \mu \mathrm{rad}_{\mathrm{rms}}$ in the rotation angle.

A PC controlled stepped ramp of $\nu$ around $\nu_{L}$ (or $\nu_{L} / 2, \nu_{L} / 3, \ldots$ ) in the open-loop configuration shows the resonance in the rotation-angle amplitude (see Fig 4 where the lock-in is referenced to the second harmonic of $\nu$ while $\nu$ is scanned around $\nu_{L} / 2$ ). Optimal magnetometer performance is obtained at a pump beam power of $3 \mu \mathrm{W}$ and a detecting laser power of $5 \mu \mathrm{W}$ at the output of the cells. The pump beam absorption is of the order of $50 \%$ and about $20 \%$ for the probe laser at the lock point. In this condition the oscillating single photodiode current is $I_{p-p}^{a c}=13 \mathrm{nA}$ at a dc current of $I^{d c}=0.8 \mu \mathrm{A}$. Thus the contrast of the resonance is about $3 \%$ for each polarimeter arm and the width is of the order of $40 \mathrm{~Hz}$ in the second harmonic detection. This width is consistent with the quality of the oscillator ( $\mathrm{Q}$ of 280, as seen from the inset) verified under identical probe conditions, with single pump pulse excitation. Increasing the probe laser power by a factor of two steepens the resonance slope by $10 \%$ but light shot noise increases at the same time. Lower probe laser power gradually decreases the slope, for example at $2.5 \mu \mathrm{W}$ the slope is $30 \%$ less.

\section{Noise and limits}

The self-oscillating mode is obtained by closing the loop so that the pump laser frequency modulation is driven by a pulse triggered by the output of the scaler (generally we use a divider by two). The overall phase shift is compensated by adjusting the delay of the pulses. In this regime the Larmor precession signal is not free from the magnetic noise in the Laboratory. Low-frequency drifts of the center-frequency readings can be seen (in the range of up to a few $10 \mathrm{~Hz}$ ) due to variations in the earth's magnetic field, ionosphere 

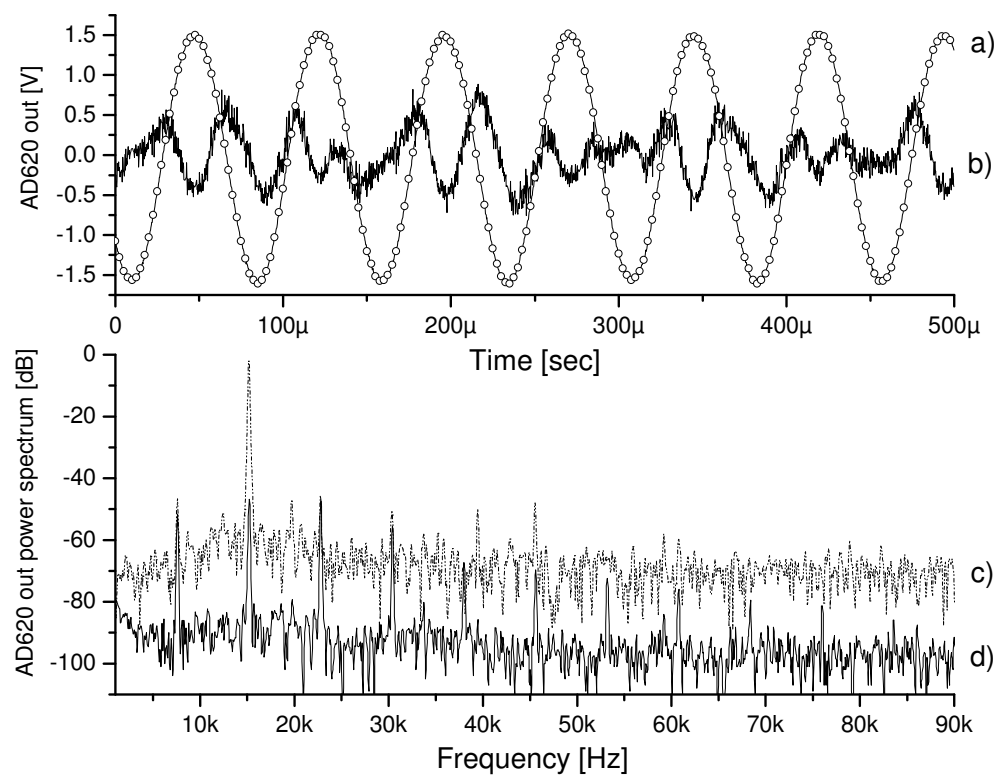

Fig. 3. Signal of one channel at the output of the instrumentation amplifier. The upper plot shows the signal vs. time (trace a) and the residual (signal minus its fundamental tone at $\nu_{L}$ ) amplified by a factor 10 (trace b). In the lower plot the power spectrum of the signal (trace c) shows a $60 \mathrm{~dB}$ contrast of the peak at $\nu_{L}$ with respect to the background noise. Trace $d$ shows details of the noise contributions. This is the power spectrum of an average of 128 traces, registered with no probe beam. Reduction of the white noise, due to averaging, makes it possible to distinguish peaks at the pump frequency and at its harmonics. It is worth noting that the harmonics at $\nu_{L}$ is almost $50 \mathrm{~dB}$ below the peak at the fundamental tone of trace $\mathrm{c}$. 


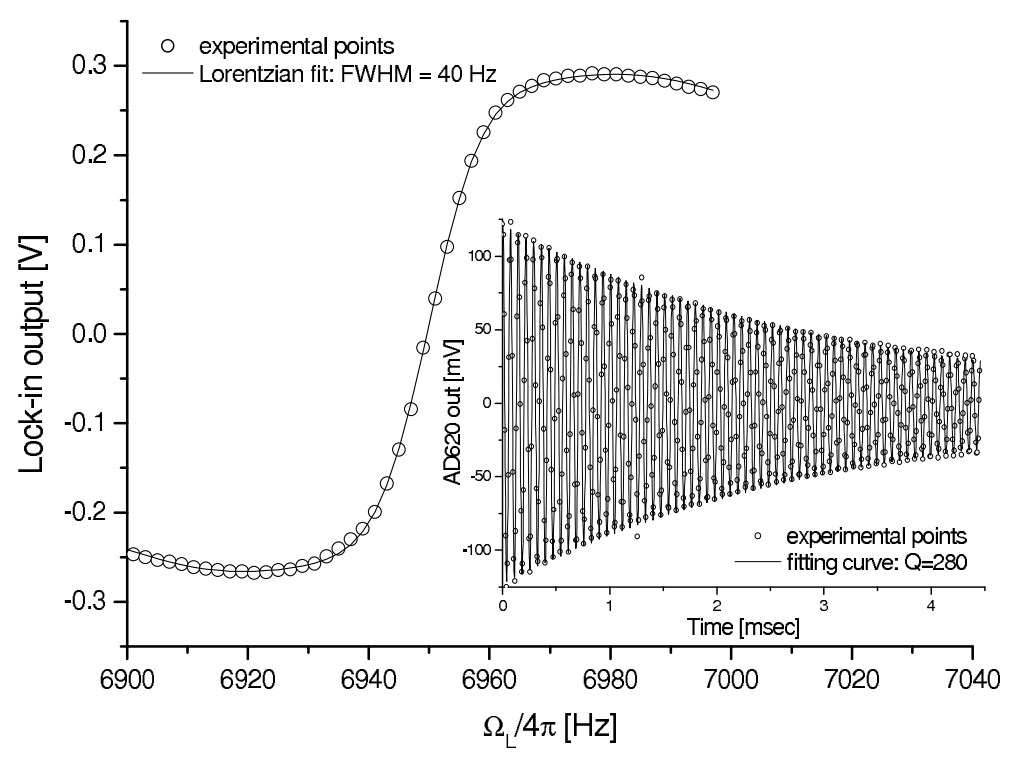

Fig. 4. Resonance in the rotation-angle amplitude registered at the output of the lock-in amplifier. The output of the instrumentation amplifier is fed into the lock-in input, whose reference signal is extracted from a pulse generator which modulates the pump laser frequency. The resonance linewidth is consistent with the quality factor inferred from the exponential decay of the amplitude after a single pump pulse (in the inset).

activity, human activity etc. The power-net contribution at $50 \mathrm{~Hz}$ and its harmonics represent the major noise contribution, ranging from a few $100 \mathrm{pT}$ up to a few nT.

In our arrangement complete compensation of the horizontal components of the dc magnetic field is performed using two orthogonal pairs of Helmholtz coils. A third pair is used to reduce the $z$ component down to about $4 \mu \mathrm{T}$. Accurate of compensation of the $\partial B_{z} / \partial y$ gradient component (the one most responsible for line broadening) is performed by means of a quadrupole field generated by a pair of magnetic dipoles symmetrically placed about $1 \mathrm{~m}$ away from the sensor. A second pair of dipoles compensates $\partial B_{z} / \partial x$, i.e. equalizes the field in the two cells. A detailed description of our dc magnetic field and gradient compensation system is given in [17].

As stressed above, the magnetometer works in an unshielded environment and can work in single or differential regimes. In single arm operation the output of the instrumentation amplifier can be monitored using a frequency counter, thus providing an absolute measure of the magnetic field.

Small relative magnetic field variations are registered in differential mode by forcing the second arm of the magnetometer with the Larmor frequency of the main arm. This allows for automatic registration of magnetic field variations with a linear dynamic range that is limited by the resonance linewidth. The fact that the two arms are only $7 \mathrm{~cm}$ away from each other makes it possible to reject a large part of the magnetic noise registered as common-mode variations. In this regime the instrumentation amplifier output of the secondary arm is demodulated with respect to the Larmor precession frequency of the main arm by means of a lock-in amplifier.

The major noise contributions calculated for each detector are as follow: the light shot noise $\left(\sqrt{2 \mathrm{qI}_{\mathrm{dc}}}=\right.$ $0.5 \mathrm{pA} / \sqrt{\mathrm{Hz}})$ of $0.62 \mathrm{pT} / \sqrt{\mathrm{Hz}}$, the Johnson noise $\left(\sqrt{4 \mathrm{RTk}_{\mathrm{B}}}=33 \mathrm{nV} / \sqrt{\mathrm{Hz}}\right)$ estimated to be $0.6 \mathrm{pT} / \sqrt{\mathrm{Hz}}$ 
and the noise of the amplifiers $(0.4 \mathrm{nA} / \sqrt{\mathrm{Hz}}, 4 \mathrm{nV} / \sqrt{\mathrm{Hz}})$ of $0.5 \mathrm{pT} / \sqrt{\mathrm{Hz}}$. The overall noise contribution from the four detectors amounts to $2 \mathrm{pT} / \sqrt{\mathrm{Hz}}$ which is consistent with the measured residual differential noise spectrum shown in Fig 5 ,

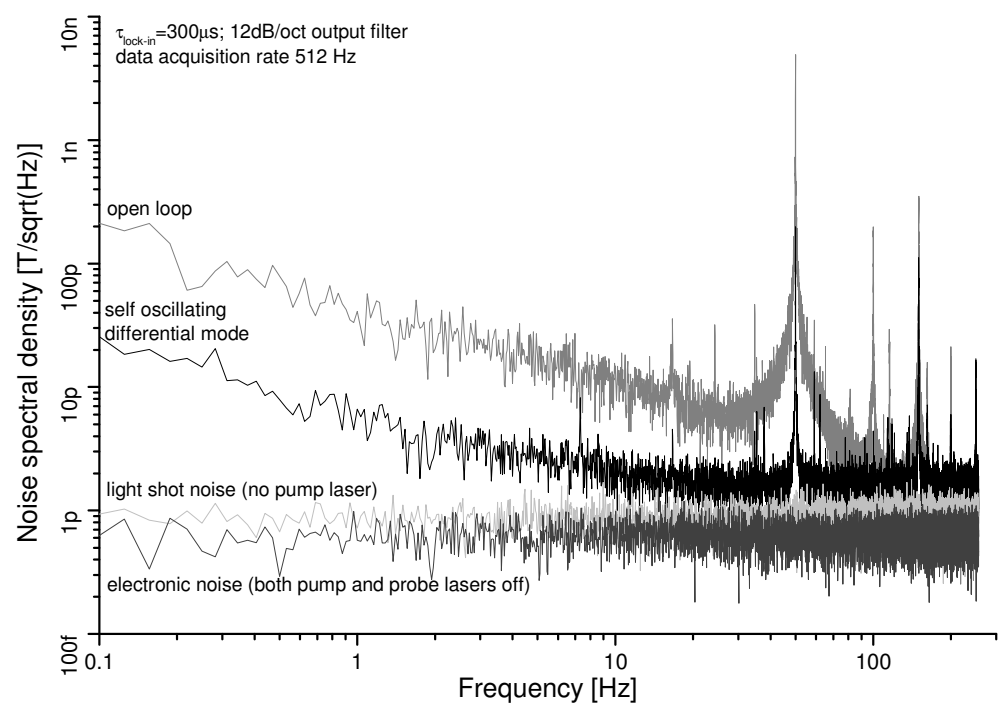

Fig. 5. Noise pattern in the open loop, self-oscillating differential arrangement, with no pump laser, and the electronic noise.

\section{Registration of water bulk magnetization}

The differential magnetometer is used to detect the bulk magnetization of tap water in the setup represented in Fig 6. The water flows through a rectangular cross-section tube $\left(6 \times 8 \mathrm{~mm}^{2}\right.$ inner size $)$ in a prepolarizing field. The prepolarizing field is generated by a set of $\mathrm{Nd}$ permanent magnets ( 1 inch cubes) aligned so as to produce a pair of $70 \mathrm{~cm}$ long magnets. The prepolarizing magnetic field strength is estimated to be $0.7 \mathrm{~T}$ at the center of the assembly (at a distance of $3.5 \mathrm{~mm}$ from the surface of the cubes). The assembly is inserted into an iron cylinder in order to reduce the external magnetic field and to increase the internal one. The water volume inside the magnet is about $30 \mathrm{ml}$ and the water flow is around $5 \mathrm{ml} / \mathrm{sec}$. Thus, the water spends at least $6 \mathrm{sec}$ in the prepolarizing magnetic field. At the output of the assembly, water flows through a capillary tube (internal diameter of $1.8 \mathrm{~mm}$ ) of $1 \mathrm{~m}$ in length, which brings it to the registration volume in $0.5 \mathrm{sec}$. The water is accumulated in a measurement bulb of $5 \mathrm{ml}$ ( $1 \mathrm{sec}$ complete refreshment time). Water passes through the bulb in a serpentine path in order to decrease its mixing in the detection volume and therefore guarantee an efficient refreshment. The center of the bulb is situated at around $1.7 \mathrm{~cm}$ from the center of the probe beam.

The water flow is modulated using a peristaltic pump which is periodically switched on and off. A typical measurement consists of a set of on-off cycles, which are necessary to average out slow drifts, and the high frequency residual noise. In each cycle, the pump-on duration is 2 sec: this time is necessary to reach a stable flow regime $(0.5 \mathrm{sec})$, transport the water to the detection region $(0.5 \mathrm{sec})$, and complete refreshment of the bulb (1 sec). The pump-off duration is $5 \mathrm{sec}$, which is necessary to let the longitudinal magnetization $\mathbf{M}$ reach complete relaxation. The $\mathbf{M}$ decay in time is detected by the magnetometer in this interval. The output of the lock-in amplifier is stored together with the pump status in time for further data averaging. 


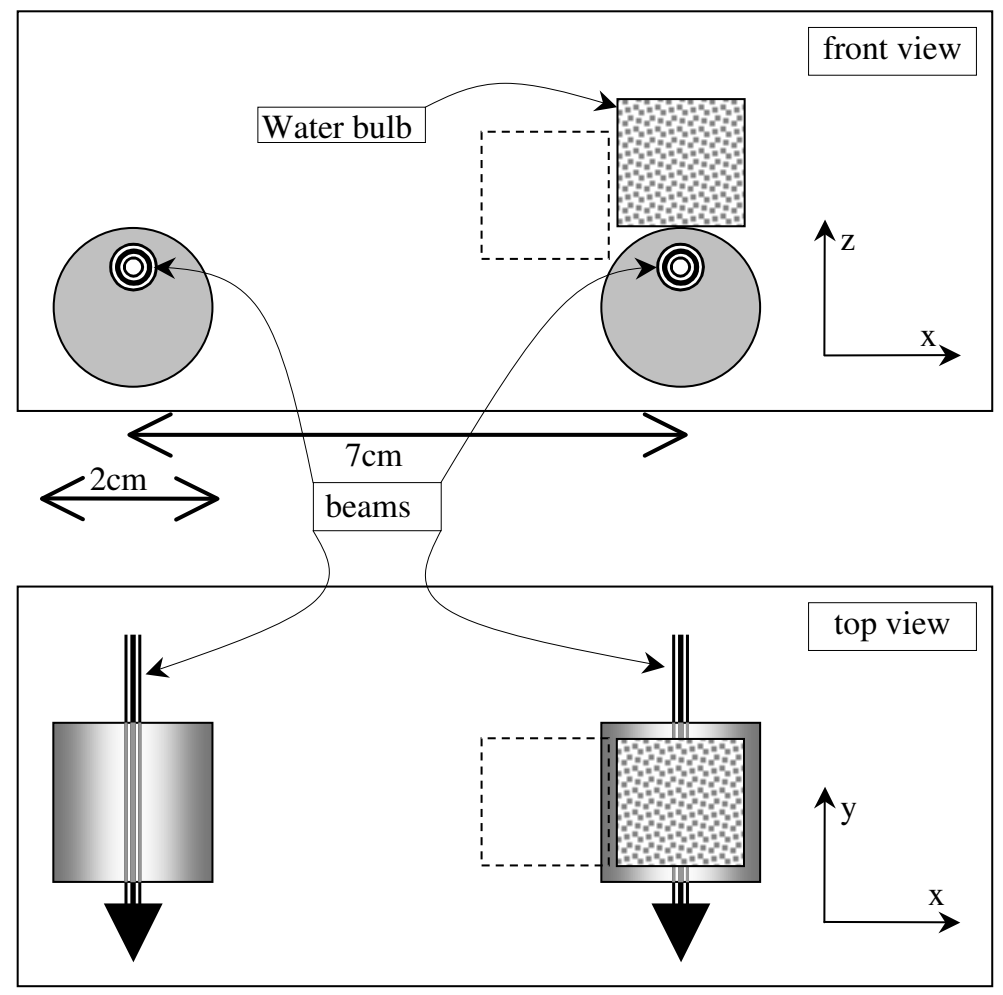

Fig. 6. Front and top views of the arrangement of the dual sensor and of the water bulb. The grey cylinders represent the Cs cells. Bulk water magnetization is measured when the bulb (dotted cube) is placed above the cell. The dashed cube represents the position of the bulb for detection of free induction decay. 
The self-oscillating operation makes the magnetometer insensitive to the slow drifts of the magnetic field (in a range of several $\mathrm{nT}$ ), thus making very long recording times possible. The stability of the system allows for autonomous recording times as long as several hours (actually they were usually limited by the duration of the working day). The average signal of $\mathbf{M}$ of 387 water pump cycles is shown in Fig.7 The estimated $T_{1}$ relaxation is of the order of $1.5 \mathrm{sec}$, as shown in the inset.

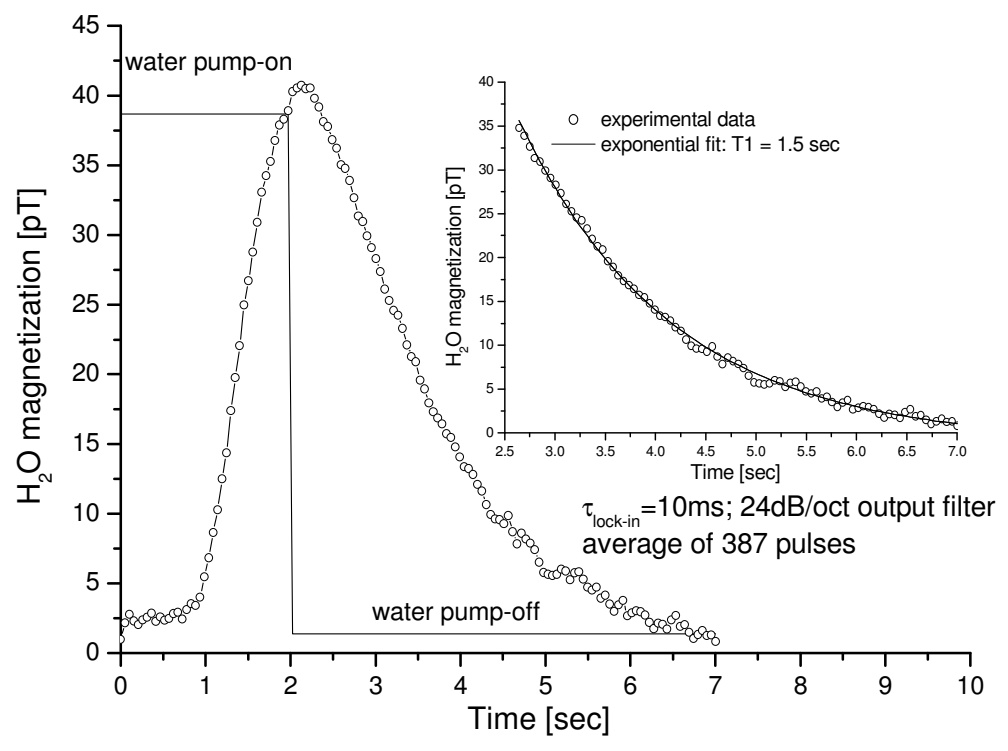

Fig. 7. Magnetic field variation in $z$ direction due to water magnetization. The signal shown is the result of an average of 387 pulses.

Water magnetization decay can also be observed via a variant of phase encoding. An inversion of the magnetization can be observed by applying $\pi$ pulse using the compensating coils ( $x$ or $y$ pair). Fig 8 shows the trace of non-perturbed $\mathbf{M}$ increase and decay, together with a trace showing its inversion when a $\pi$ pulse is applied at the end of the pump-on interval after $0.5 \mathrm{sec}$ delay. The $\pi$ pulse is composed of 8 periods of sine waveform whose frequency is automatically determined by reading the current lock-in reference frequency (the atomic Larmor frequency of the main arm) and scaling it by the ratio between the atomic and nuclear gyromagnetic factors.

The response of the self-oscillating magnetometer at higher frequencies is evaluated by detecting the free induction decay (FID) of the nuclear spins. In this case the magnetometer works in single arm mode (only using the arm closest to the water bulb). In order for the FID to contribute with a non-vanishing component parallel to the bias magnetic field $B_{z}$ (i.e. to produce a signal detectable by a scalar magnetometer), the sample is displaced by about $45^{\circ}$ with respect to the direction of the bias field (see Fig, 6). The geometrical constraints (larger distance and angular spread) make the signal noticeably weaker than in the dc case (the maximum dc bulk magnetization detected is of the order of $30 \mathrm{pT}$ ), so that accurate signal processing is necessary to extract it. In the case of Fig 9 the procedure consists in acquiring a set of traces (direct digitization of the instrumentation amplifier output) starting at the end of the $\pi / 2$ pulse. The signal is digitized and numerically demodulated with the carrier frequency (at $\nu_{L} \approx 13 \mathrm{kHz}$ ), which is evaluated for each single trace. Following a $200 \mathrm{~ms}$ delay, which is necessary to stop the water flow completely, the pump-off triggers the $\pi / 2$ pulse, at the end of which a trace acquisition starts. The data sets are averaged 


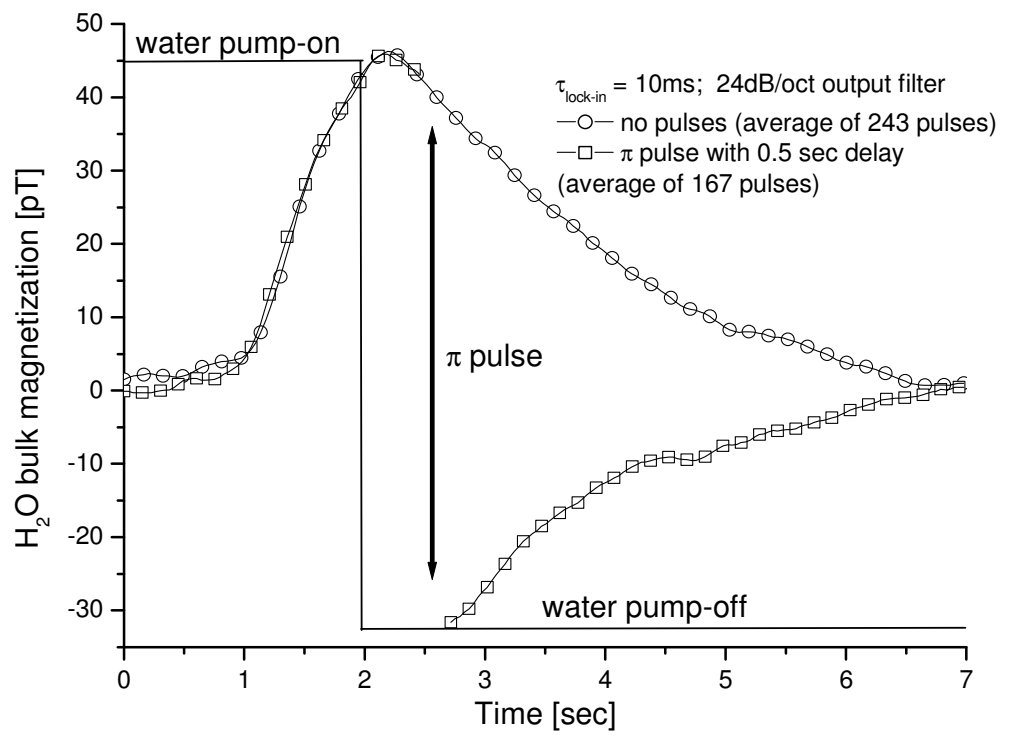

Fig. 8. Magnetic field due to water flowing in the detection region (circles) and following a $\pi$ pulse (squares). The data are recorded with a time constant of $10 \mathrm{~ms}$ and a $24 \mathrm{~dB} /$ oct output filter. The $\pi$ pulse is applied using $x$ direction compensating coils.

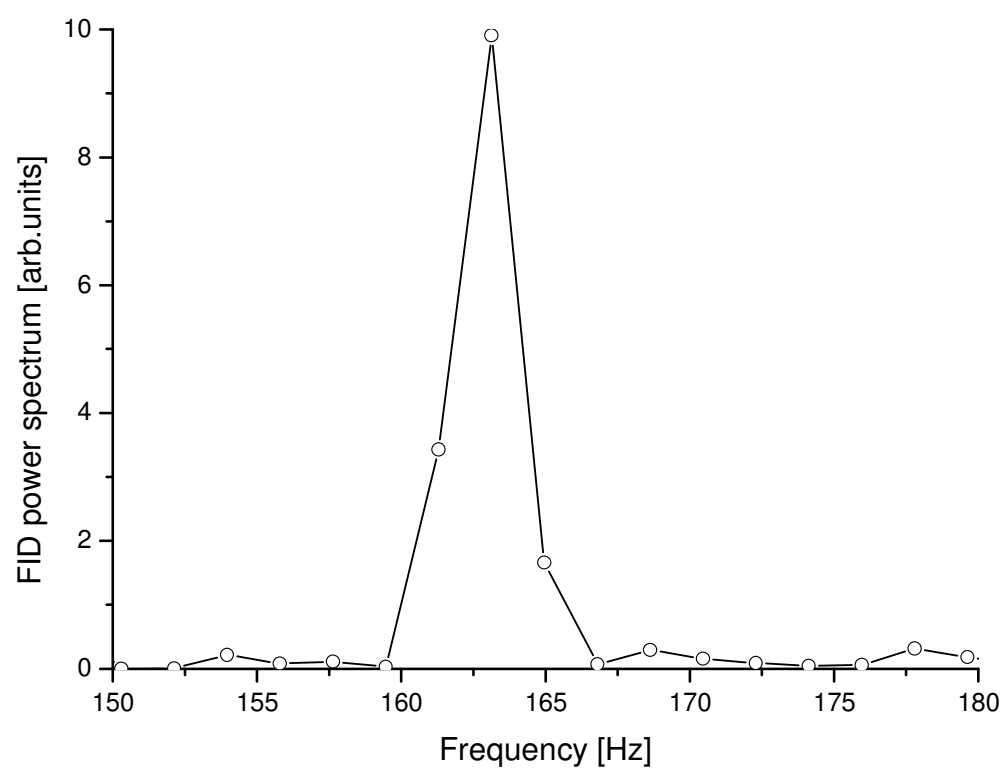

Fig. 9. Power spectrum of the magnetic field demodulated with the atomic Larmor frequency. The peak at $163 \mathrm{~Hz}$ corresponds to the FID following the $\pi / 2$ pulse applied with the $x$ pair of compensating coils. The signal is acquired at $30 \mathrm{kS} / \mathrm{s}$ and is an average of 43 pulses. 
and analyzed in order to reduce the amplitude of the noise components having a random phase with respect to the trigger and to highlight the nuclear Larmor precession at $163 \mathrm{~Hz}$. Deterministic noise contributions at $50 \mathrm{~Hz}$ and its harmonics (up to the $3^{\text {rd }}$ harmonic) are estimated and subtracted prior to evaluation of the power spectrum.

\section{Conclusions}

We have demonstrated a two-arms and two-beams self-oscillating magnetometer based on nonlinear magnetooptical rotation. The use of a non-modulated probe beam provides a clear Larmor precession signal and the use of sub-harmonic excitation prevents fake signals in the loop. The optical magnetometer reaches a sensitivity of $2 \mathrm{pT} / \sqrt{\mathrm{Hz}}$ in unshielded environment. The differential sensor makes the optical magnetometer robust and gives it a high dynamic range when working in self-oscillating regime. It also brings the further advantage of long operating times and thus the possibility of significant noise reduction when using, for example, an off-line averaging procedure. The sensor was used for the NMR registration, detecting both the dc magnetization and the nuclear spins precession of remotely polarized hydrogen nuclei in water samples. The low-field operation and the corresponding low precession frequency of the nuclei would facilitate nuclear spin manipulation via non-adiabatic magnetic pulses. It should be emphasized that such high sensitivity optical magnetometry offers the possibility of NMR registration using relatively low prepolarizing magnetic fields.

\section{Acknowledgments}

The authors would like to acknowledge very useful suggestions about the implementation of the self-oscillating set-up with D. Budker, encouraging discussions with A. Cassarà about low magnetic field NMR detection, and Emma Thorley for her kind collaboration in improving the manuscript. G. B., V. B., Y. D. and L. M. would like to acknowledge the financial support of the Monte dei Paschi Foundation (ref. number 27285).

\section{References}

1. H. G. Dehmelt, "Modulation of a light beam by precessing atoms", Phys. Rev. 105, 1924-1925 (1957).

2. W. Bell, A. L. Bloom, "Optical detection of magnetic resonance in alkali metal vapor", Phys. Rev. 107, 1559-1565 (1957).

3. A. L. Bloom, "Principles of operation of the rubidium vapor magnetometer", Appl. Opt. 1, 61-68 (1962).

4. J. Dupont-Roc, S. Haroche and C. Cohen-Tannoudji, "Detection of very weak magnetic fields $\left(10^{-9}\right.$ gauss) by $\mathrm{Rb}^{87}$ zero-field level crossing resonances", Phys. Lett. 28A, 638-639 (1969).

5. C. Cohen-Tannoudji, J. Dupont-Roc, S. Haroche and F. Laloe, "Detection of the static magnetic field produced by the oriented nuclei of optically pumped 3He gas", Phys. Rev. Lett. 22, 758-760 (1969).

6. E. B. Alexandrov, M. V. Balabas, A. S. Pasgalev, A. K. Vershovskii and N. N. Yakobson, "Doubleresonance atomic magnetometers: from gas discharge to laser pumping", Laser Phys. 6, 244-251 (1996).

7. R. Wynands and A. Nagel, "Precision spectroscopy with coherent dark state", Appl. Phys. B 68, 1-25 (1999).

8. D. Budker, V. Gawlik, D. F. Kimball, S. M. Rochester, V. V. Yashchuk and A. Weis, "Resonant nonlinear magneto-optical effects in atoms", Rev. Mod. Phys. 74, 1153-1201 (2002).

9. V. Acosta, M. P. Ledbetter, S. M. Rochester, D. Budker, D. F. Kimball, D. C. Hovde, W. Gawlik, S. Pustelny and J. Zachorowski, "Nonlinear magneto-optical rotation with frequency-modulated light in the geophysical field range", Phys. Rev. A 73, 053404 (2006).

10. M. V. Romalis and D. Budker, "Optical magnetometry", Nature Physics 3, 227-234 (2007). 
11. I. M. Savukov, S. K. Lee and M. V. Romalis, "Optical detection of liquid state NMR", Nature (London) 442, 1021-1024, (2006).

12. I. M. Savukov and M. V. Romalis, "NMR detection with an atomic magnetometer", Phys. Rev. Lett. 94, 123001 (2005).

13. Sh. Xu, V. V. Yashchuk, M. H. Donaldson, S. M. Rochester and D. Budker, "Magnetic resonance imaging with an optical atomic magnetometer", PNAS 103, 12668-12671 (2006).

14. I. M. Savukov, S. J. Seltzer and M. V. Romalis, "Detection of NMR signals with a radio-frequency atomic magnetometer", JMR 185, 214-220 (2007).

15. M. P. Ledbetter, I. M. Savukov, D. Budker, V. Shah, S. Knappe, J. Kitching, D. J. Michalak, S. Xu and A. Pines, "Zero-field remote detection of NMR with a microfabricated atomic magnetometer", PNAS 105, 2286-2290 (2008).

16. C. Andreeva, G. Bevilacqua, V. Biancalana, S. Cartaleva, Y. Dancheva, T. Karaulanov, C. Marinelli, E. Mariotti and L. Moi, "Two-color coherent population trapping in a single Cs hyperfine transition, with application in magnetometry", Appl. Phys. B 76, 667-675 (2003).

17. J. Belfi, G. Bevilacqua, V. Biancalana, S. Cartaleva, Y. Dancheva and L. Moi, "Cesium coherent population trapping magnetometer for cardio signal detection in an unshielded environment", J. Opt. Soc. Am. B 24, 2357-2362 (2007).

18. D. Suter and J. Mlynek, "Laser excitation and detection of magnetic resonance", Advances in Magnetic and Optical Resonances 16, 1-83 (1991).

19. N. Beverini, P. Violino and F. Strumia, "Optical pumping of Caesium in the presence of heavy noble gases", Zeitschrift für Physik 265, 189-196 (1973).

20. M. T. Graf, D. F. Kimball, S. M. Rochester, K. Kerner, C. Wong, D. Budker, E. B. Alexandrov, M. V. Balabas and V. V. Yashchuk, "Relaxation of atomic polarization in paraffin-coated cesium vapor cells", Phys. Rev. A 72, 023401 (2005).

21. J. S. Guzman, A. Wojciechowski, J. E. Stalnaker, K. Tsigutkin, V. V. Yashchuk and D. Budker, "Nonlinear magneto-optical rotation, Zeeman and hyperfine relaxation of potassium atoms in a paraffin-coated cell", Phys. Rev. A 74, 053415 (2006).

22. J. M. Higbie, E. Corsini and D. Budker, "Robust, high-speed, all-optical atomic magnetometer", Rev. Sci. Instr. 77, 113106 (2006).

23. J. Vanier and C. Audoin, "The Quantum Physics of Atomic Frequency Standards", Adam Hilger Eds. Bristol and Philadelphia, Chap.3, p.410 (1989).

24. J. Belfi, G. Bevilacqua, V. Biancalana, Y. Dancheva and L. Moi, "All-optical sensor for automated magnetometry based on coherent population trapping", J. Opt. Soc. Am. B 24, 1482-1489 (2007).

25. P. D. D. Schwindt, L. Hollberg and J. Kitching," Self-oscillating rubidium magnetometer using nonlinear magneto-optical rotation", Rev. Sci. Instrum. 76, 126103 (2005).

26. We use OSD15-5T (Centronic) photodiodes with a sensitivity of $0.45 \mathrm{~A} / \mathrm{W}$ and $5 \mathrm{nA}$ dark current. Each photo-current is amplified using an OP484 chip (Analog devices), which contains four low-noise operational amplifiers (OAs). One $\mathrm{OA}$ is configured as the transimpedance amplifier, with a feedback impedance made by a $68 k \Omega$ resistor in parallel with a $47 \mathrm{pF}$ capacitor. Another OA is used as a dc voltage amplifier, while the two remaining OAs are used as cascaded bandpass amplifiers (each one has an ac coupled input and a RC parallel in its feedback, with cutoff frequencies set at $10 \mathrm{kHz}$ and $40 \mathrm{kHz}$, respectively). 